\title{
A retrospective clinical observation of long-term survival in patients after prolonged mechanical ventilation
}

Chienhsiu Huang ( $\sim$ hgssport@yahoo.com.tw)

\section{Research}

Keywords: prolonged mechanical ventilation, respiratory care center, five-year survival rate

Posted Date: January 21st, 2020

DOl: https://doi.org/10.21203/rs.2.21403/v1

License: (c) (1) This work is licensed under a Creative Commons Attribution 4.0 International License.

Read Full License 


\section{Abstract}

\section{Background}

The 1-year survival rate of patients on prolonged mechanical ventilation was $29-42 \%$ before 2000 . By 2012 , Carson reported the 1 -year survival rate of prolonged mechanical ventilation patients was $52 \%$, an unsatisfactory improvement in survival time. We explored the long-term survival rate of prolonged mechanical ventilation patients at our hospital, 2012-2017.

\section{Methods}

We analyzed retrospectively data from our respiratory care center patients to investigate the discharge status, long-term survival rate, and cause of death. We also compared the 5-year survival rates of prolonged mechanical ventilation patients and our hospital lung cancer patients.

\section{Results}

Five hundred seventy-four patients were admitted to respiratory care center during the study period. The long-term follow-up data of 403 prolonged mechanical ventilation, including 186 patients died in the hospital, 157 discharged prolonged mechanical ventilation patients, and 60 respiratory care ward patients until October 30, 2018. The 1-year survival rate of all prolonged mechanical ventilation patients, discharged prolonged mechanical ventilation patients, and respiratory care ward patients were $24.3 \%$, $50.3 \%$, and $31.7 \%$, respectively. The 5 -year survival rates of all prolonged mechanical ventilation patients, discharged prolonged mechanical ventilation patients, and respiratory care ward patients were $18.1 \%$, $40.05 \%$, and $11.7 \%$, respectively. Successful weaning from mechanical ventilation was a key factor in the long-term survival of prolonged mechanical ventilation patients. The 5-year survival rate of discharged prolonged mechanical ventilation patients was better than that of all lung cancer patients. The 5-year survival rates of all prolonged mechanical ventilation patients and respiratory care ward patients were similar to that of stage IV lung cancer patients.

\section{Conclusions}

Prolonged mechanical ventilation patients had the worst long-term survival rate of all patients with chest diseases in our hospital. Successful weaning from the mechanical ventilator was most important factors for improving long-term outcomes in prolonged mechanical ventilation patients.

\section{Introduction:}

"The Trial Plan for National Public Health Insurance Ventilator Dependent Patients Comprehensive Care System" has been promoted in Taiwan since July 2000 [1]. The program covers mechanical ventilator care in the following settings: intensive care unit, respiratory care center (RCC), respiratory care ward (RCW), and home care service. We retrospectively studied 574 RCC patients and reported the comprehensive clinical experience of patients receiving prolonged mechanical ventilation (PMV) in 2019 
[2]. Carson's study shows that patients requiring PMV have poor long-term outcomes which have not improved significantly since 2006 [3]. Damuth and colleagues reported a 1-year survival rate in all weaned units in acute care hospitals of $45.2 \%$ [4]. We sought to explore the long-term survival rate of PMV patients in one RCC over six years.

\section{Methods:}

We retrospectively studied the medical records of 574 patients admitted to the RCC from January 2012 to December 2017 and collected their age, gender, comorbidities, cause of respiratory failure, RCC discharge status, weaning status, and long-term survival rate. For those who died of pneumonia, the relevant pathogens were also recorded.

Ethics approval and consent to participate:

Our study was a retrospective review and analysis of medical records. Information was de-identified before use and the research risk was identified as minimal. The project was approved by the Buddhist Dalin Tzu Chi General Hospital Research Ethics Committee (Approved IRB No.: B10802009), which exempted the study from requiring informed consent from participants.

Definitions:

A PMV patient was defined as one who used a mechanical ventilator for at least six hours daily for 21 or more consecutive days. A successfully-weaned patient was defined as one who was independent from the ventilator for five consecutive days and nights. These patients were transferred to the ward. The unsuccessfully-weaned ventilator patients included those who died in the RCC, hospice patients in the RCC, and ventilator-dependent patients. Ventilator-dependence was defined as those who could not be weaned from the ventilator and were transferred to the RCW for long-term ventilator support. The hospital mortality rate was determined by the number of patients who died in the hospital (including those who died in the RCC, successfully-weaned patients who died in the ward before discharge, and hospice patients) divided by the total number of patients admitted to the RCC.

The causes of acute respiratory failure leading patients to require PMV:

We classified 11 causes of acute respiratory failure leading patients to require PMV, as follows: (1) pneumonia, (2) intracranial hemorrhage, (3) sepsis, (4) postoperative condition, (5) chronic obstructive pulmonary disease (COPD), (6) cardiac disease (including decompensated heart failure, coronary atherosclerotic disease, and acute myocardial infarction), (7) underlying malignant disease, (8) cervical spine disease, (9) post Cardio-Pulmonary-Cerebral-Resuscitation hypoxic encephalopathy, (10) cerebral infarction, and (11) miscellaneous causes.

Comorbidities:

The number of comorbidities was assessed. These included: cardiovascular disease (such as essential hypertension, decompensated heart failure, coronary atherosclerotic disease, acute myocardial 
infarction), chronic lung disease (such as COPD, asthma, bronchiectasis, interstitial lung disease), chronic kidney disease (CKD) (not requiring dialysis), end-stage renal disease (ESRD) (requiring dialysis before admission), neurologic disease (such as bed-ridden stroke survivors, or those with dementia or parkinsonism), chronic liver disease (such as chronic hepatitis, liver cirrhosis), metabolic disease (such as diabetes mellitus or other endocrine disorders requiring treatment), malignant diseases, and miscellaneous diseases. Long-term 5-5-

Five years survival rate:

We studied the 5-year survival rate of all PMV patients, discharged PMV patients, and RCW patients. This 5-year survival rate of PMV patients was also compared with that of our hospital lung cancer patients during the same period.

Cause of death in PMV patients:

We collected the cause of death of all PMV patients. If the cause of death was pneumonia, we also collect the pathogens of pneumonia.

Statistical analysis:

Continuous variables were expressed as mean \pm standard deviation or median (range), whereas categorical variables were expressed as frequencies and percentage. Differences in baseline characteristics, causes of respiratory failure, and number of comorbidities were evaluated using the Student's t-test for continuous variables and Pearson chi-square tests for categorical variables. Univariate analysis was used to analyze the association of each variable with 1-year survival in all PMV patients, discharged PMV patients, and RCW patients. Multivariate stepwise logistic regression models were used to assess the factors related to 1-year survival in all PMV patients, discharged PMV patients, and RCW patients. The Kaplan-Meier method used to estimate the cumulative probability of survival as a function of the number of months in all PMV patients, discharged PMV patients, RCW patients, lung cancer patients, and stage IV lung cancer patients. The log-rank test was used to compare survival rates between groups. Cox proportional hazards models were used to determine the relationship of various factors to the survival rates of the five groups of patients. All statistical analyses were conducted using the statistical package SPSS for Windows, Version 17.0 (SPSS Inc., Chicago, IL) and a P value $<0.05$ was considered to show statistical significance.

\section{Results:}

A total of 574 patients were admitted to the RCC during the six years of the study. Of these, 357 patients $(62.2 \%)$ were men and 217 (37.8\%) were women. The mean age was 72.4 years. Three hundred and ninety-one patients (68.1\%) were successfully weaned from the ventilator, and $83(14.5 \%)$ unsuccessfullyweaned with ventilator-dependence patients were transferred to the RCW. In all, 186 patients died in the hospital, including 95 RCC patients, 5 hospice patients, and 86 successfully-weaned patients who expired before hospital discharge. The hospital mortality rate was $32.4 \%$. 
The long-term follow-up data of $403 \mathrm{PMV}$ patients included 186 who died in the hospital, 157 discharged PMV patients, and 60 RCW patients followed until October 30, 2018. The 1-year survival rate of all PMV patients was $24.3 \%$. The mean age of all PMV patients who survived for more than one year was significantly different from that of those who survived less than one year (67.4 years vs. 75.0 years, $P<$ 0.001). In all PMV patients, those with no comorbidity $(P=0.002$, odds ratio $[O R]=3.645)$ and successfully weaned patients $(P<0.001, O R=3.492)$ had better 1 -year survival rates. Patients older than 75 years $(P=0.005, O R=0.464)$, those with ESRD $(P=0.040, O R=0.275)$, and those with four or more comorbidities $(P=0.021, O R=0.180)$ had poorer 1-year survival rates [2]. The 1-year survival rate of discharged PMV patients was $50.3 \%$. The mean age of discharged PMV patients who survived for more than one year was significantly different from that of those who survived less than one year (66.69 years vs. 78.04 years, $P<0.001$ ). Discharged $P M V$ patients older than 75 years had a poorer 1 -year survival rate $(P=0.001, O R=0.308)$, as did those with four or more comorbidities $(P=0.031, O R=0.094)$. The 1-year survival rate of RCW patients was $31.7 \%$. The mean age of RCW patients who survived for more than one year was no significantly different from that of those who survived less than one year (72.36 years vs 69.78 years, $P=0.513)$. Multivariate analysis showed no significant difference in the clinical variables of RCW patients who did or did not survive one year or more.

Pneumonia was the leading cause of death in PMV patients. In all, 159 PMV patients (39.5\%) died due to pneumonia, including 106 patients with detected pneumonia pathogen. Sixty percent of detected pneumonia pathogens were polypathogenic. The most-frequent pneumonia pathogens in PMV patients were, in order, Acinetobacter baumannii (29.7\%), Pseudomonas aeruginosa (17.7\%) and Klebsiella pneumonia (12.0\%). Carbapenems-resistant gram-negative bacilli represented $35.4 \%$ of the identified pneumonia pathogens, especially carbapenem-resistant Acinetobacter baumannii (CRAB) (25.7\%). The 6month, 1-year, 2-year, and 5-year mortality rates of all PMV patients were $68.2 \%, 75.9 \%, 79.7 \%$, and 81.9\%, respectively. The 6-month, 1-year, 2-year, and 5-year mortality rates of discharged PMV patients were $31.8 \%, 49.7 \%, 56.1 \%$, and $59.95 \%$, respectively. The 6-month, 1-year, 2-year, and 5-year mortality rates of RCW patients were $60.0 \%, 68.3 \%, 80.0 \%$, and $88.3 \%$, respectively (Fig. 1).

Kaplan-Meier analysis of all PMV patients, discharged PMV patients, and RCW patients showed that discharged PMV patients had the best 5-year survival rate. The 5-year survival rate did not significantly differ between all PMV patients and RCW patients. Compared to our hospital lung cancer patients, discharged PMV patients had a better 5-year survival rate than all lung cancer patients and stage IV lung cancer patients. All PMV patients had a worse 5-year survival rate than all lung cancer patients. The 5year survival rate did not significantly differ between all PMV patients and stage IV lung cancer patients. RCW patients had a worse 5-year survival rate than all lung cancer patients. The 5-year survival rate did not significantly differ between RCW patients and stage IV lung cancer patients. (Fig. 2 and Fig. 3).

\section{Discussion:}

Overall 1-year survival rate of all PMV patients: 
Dettmer identified six factors (increasing age, vasopressor use, thrombocytopenia, acute kidney injury, inability to wean from the ventilator, and preexisting kidney injury) that had a strong association with mortality in PMV patients [5]. The literature reports that the 1-year survival rate of PMV patients ranges 37.2-61\% (Table 1) [6-14]. Older age, failure to wean, and a higher Acute Physiology, Age, Chronic Health Evaluation II (APACHE II) score were associated with a poor 1-year survival rate. In Carson 's study [11], patients older than 65 years had a poorer 1-year survival rate than younger patients. In contrast, we found that being older than 75 years was associated with a poor 1-year survival rate. Carson and colleagues reported that thrombocytopenia, requiring vasopressors, and ESRD were three other factors related to a poor 1-year survival rate [11]. Huang's study revealed that congestive heart failure, ESRD, malignancy, and liver cirrhosis were factors related to a poor 1-year survival rate, but having a tracheostomy was related to an excellent 1-year survival rate [14]. Lin and colleagues found that young age and absence of liver cirrhosis were associated with a good 1-year survival rate [13]. In our series, having ESRD or four or more comorbidities was associated with a poor 1-year survival rate. Patients with no comorbidities and those successfully weaned from ventilation had an excellent 1-year survival rate. Despite our high rate of successful weaning (68.1\%), our PMV patients had an abysmal 1-year survival rate (24.3\%), which was due to our high hospital mortality rate. The factors influencing the 1-year survival rate of PMV patients agreed with those reported in the literature.

Table 1: The one-year survival of all PMV patients 


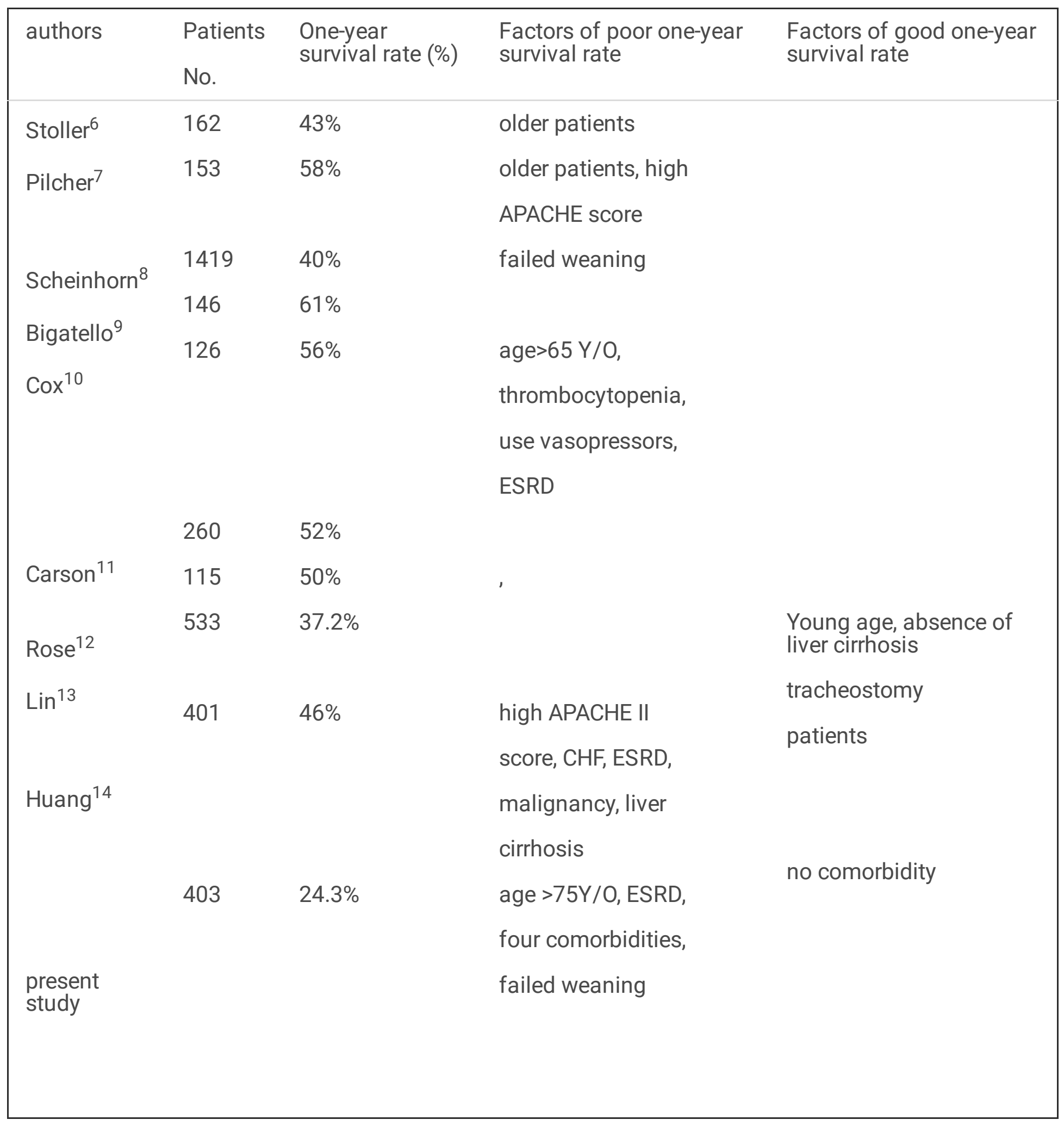

The 1-year survival rate of discharged PMV patients:

The articles which discussed the 1-year survival rate of discharged PMV patients are listed in Table 2 [15-18]. The 1-year survival rate of discharged PMV patients ranged 44.6-66.9\%. The factors related to poor 1-year survival rate in these patients were older age, high APACHE II score, high Simplified Acute 
Physiology Score, and low body mass index. The 1-year survival rate of our discharged PMV patients was $50.3 \%$. Age $>75$ years and having four or more comorbidities were associated with a poor 1 -year survival rate. Davies reported that the nocturnal use of noninvasive ventilation (NIV) was associated with an excellent 1-year survival rate [17]. This clinical experience of PMV patients was the only strategy suggested in the literature to improve the survival rate.

Table 2: The one-year survival of PMV discharge patients

\begin{tabular}{|c|c|c|c|c|}
\hline authors & $\begin{array}{l}\text { Patients } \\
\text { No. }\end{array}$ & $\begin{array}{l}\text { One-year } \\
\text { survival } \\
\text { rate (\%) }\end{array}$ & $\begin{array}{l}\text { Factors of poor one-year survival } \\
\text { rate }\end{array}$ & $\begin{array}{l}\text { Factors of good } \\
\text { one-year survival } \\
\text { rate }\end{array}$ \\
\hline Schonhofer ${ }^{15}$ & 293 & $49.4 \%$ & failed weaning & $\begin{array}{l}\text { young age, low } \\
\text { APACHE II score }\end{array}$ \\
\hline $\mathrm{Su}^{16}$ & 244 & $44.6 \%$ & & \\
\hline Davies $^{17}$ & 458 & $65.0 \%$ & $\begin{array}{l}\text { failed weaning, } \\
\text { old age, }\end{array}$ & $\begin{array}{l}\text { nocturnal use of } \\
\text { NIV }\end{array}$ \\
\hline Jubran $^{18}$ & 315 & $66.9 \%$ & $\begin{array}{l}\text { High SAPS score, High APACHII, } \\
\text { low body-mass index, failure } \\
\text { weaning } \\
\text { age }>75 Y / 0 \text {, }\end{array}$ & \\
\hline present study & 157 & $50.3 \%$ & four comorbidities, & \\
\hline
\end{tabular}

The 1-year survival rate of RCW patients:

Very few articles address the 1-year survival rate in RCW patients because RCW is a unique chronic longterm care phase in Taiwan. Su and colleagues reported a 23.6\% 1-year survival rate of RCW patients, which was lower than that of discharged PMV patients [16]. We found a 1-year survival rate of RCW patients of 31.7\%; this rate, too, was lower than that of discharged PMV patients. Jubran and colleagues reported a 1-year survival rate of $16.4 \%$ for ventilator-attached patients (similar to our RCW patients) and $66.9 \%$ for ventilator-detached patients (similar to our discharged PMV patients) [18]. Clearly, weaning from the ventilator is important for long-term survival in PMV patients.

Causes of death in PMV patients:

Pneumonia was the leading cause of death in our PMV patients. Carbapenems-resistant Gram-negative bacilli make up $35.4 \%$ of detected pneumonia pathogens, especially CRAB. Chien showed that the rise of multidrug-resistant $A$. baumannii was significantly correlated with previous exposure to piperacillin/tazobactam and imipenem. The multidrug-resistant microorganisms that cause pneumonia 
have been associated with increased 6-month mortality in PMV patients [19]. Another Taiwan's study showed that the prior use of imipenem, meropenem, piperacillin/tazobactam, or fourth-generation cephalosporins was an the independent risk factor for extensively drug-resistant A. baumannii hospitalacquired infections [20]. Having a carbapenems-resistant Gram-negative bacillus infection was fatal in our PMV patients. We must pursue the prudent use of broad-spectrum antibiotics, especially the strict use of carbapenems in our hospital.

Declining mortality rate in PMV patients:

The declining mortality rate differed among all PMV patients, discharged PMV patients, and RCW patients. All PMV patients showed a steep decline in mortality within the first three months, a slower decline in the second year, and a subsequent plateau in mortality after two years. Discharged PMV patients showed a steady decline in mortality rate within the first year, a slower decline in the second year, and a subsequent plateau after two years. RCW patients had a very steep decline in mortality within the first six months, a slower decline in the second year, and a subsequent plateau in decline after two years. RCW patients displayed a faster decline in mortality rate than discharged PMV patients, with the same 2year mortality rate as for all PMV patients. This finding means that, despite the long-term invasive mechanical ventilator support they received, RCW patients did not enjoy a better long-term survival rate. Again, successful weaning from the mechanical ventilator is a key factor in the long-term outcome of PMV patients. In Stoller's study, the mortality rate of patients discharged from the weaning unit steeply declined in the first two years (68\%), with a slower decline after that (5-year mortality rate, $81 \%$ ) [6]. The mortality rates and trends in decline of our PMV patients were similar to those reported in the series by Stollers. Aboussouan and colleagues reported that $55 \%$ of patients (both weaned and not weaned) died within the first year after discharge, with a subsequent plateau in mortality such that $40 \%$ remained alive by the second and third years [21]. In our series of weaned and not weaned patients, the 1-year, 2-year, 3year, and 5-year survival rates were $45,2 \%, 37.3 \%, 35.0 \%$, and $32.3 \%$, respectively, slightly less than the survival rates reported by Aboussouan.

Long-term survival in PMV patients and lung cancer patients:

Few studies have discussed 5-year survival in PMV patients. In our series, the 5-year survival rate was $18.1 \%$, comparable to the $19 \%$ reported by Stoller [6]. The Kaplan-Meier curve is mostly used for 5-year survival analysis of cancer patients. Therefore, we used the 5-year survival rate of lung cancer patients in our Cancer Committee database as a reference to better understand the 5-year survival rate of PMV patients. Lung cancer is commonly believed to be the most severe disease in chest medicine. Therefore, we compared the 5-year survival rates of PMV patients and lung cancer patients. To our surprise, only the 5-year survival rate of discharged PMV patients was better than that of all lung cancer patients. However, all PMV patients and RCW patients had worse 5-survival rates than did all lung cancer patients. The 5year survival rates of these two groups were similar to that of stage IV lung cancer patients. Of course, different diseases and different patient groups are incomparable. However, comparing the 5-year survival 
rate of PMV patients in the context of that of lung cancer patients at our hospital urges an immediate and forceful effort to improve the 5-year survival rate of PMV patients.

Improving the long-term survival of PMV patients:

There are many potential lines of attack to improving the long-term survival of PMV patients. First, the leading cause of death in PMV patients was pneumonia. This finding indicates the need to focus aggressively on pneumonia prevention strategies for PMV patients. Secondly, a carbapenems resistant Gram-negative bacilli infection was fatal in our PMV patients. An appropriate response to this threat should include a prudent use of broad-spectrum antibiotics active against Gram-negative bacteria, especially carbapenems. Third, failure to liberate from the ventilator was a significant factor in the poor long-term survival of PMV patients. Efforts are urgently needed to increase the liberation rate and thereby improve the survival rate of PMV patients. Each RCC in Taiwan should set up a standard ventilator weaning guideline to assist in this effort. Finally, Davies et al. reported that their patients who received nocturnal NIV had an excellent 1-year survival rate [17]. For PMV patients who cannot be weaned from the invasive mechanical ventilator, those who are suitable should be changed to NIV therapy. Thus, each RCW can take positive steps to increase the survival rate of its PMV patients.

Limitations of our study

Our study had some limitations. We did not collect patient's laboratory data, respiratory parameters, APACHE II score, Glasgow Coma Scale, or other variables related to PMV patient's long-term survival rate. Therefore, we could not analyze the impact of these factors. The literature reports that both a high APACHE II score and thrombocytopenia are associated with poor 1-year survival rate in all PMV patients. Although we made some conclusion about the long-term survival rate in all PMV patients, discharged PMV patients, and RCW patients, these results are concluded only from our retrospective single-unit investigation. Because RCW is a chronic ventilator-dependent ward unique to Taiwan, few articles on it exist in the literature. The popularity of RCWs in Taiwan lead us to expect the publication of future articles related to the clinical experience of this unit.

\section{Conclusion:}

The 1-year survival rates of all PMV patients, discharged PMV patients, and RCW patients were $24.3 \%$, $50.3 \%$, and $31.7 \%$, respectively. The 5 -year survival rates of all PMV patients, discharged PMV patients, and RCW patients were $18.1 \%, 40.05 \%$, and $11.7 \%$, respectively. The 5 -year survival rate of discharged PMV patients was better than that of lung cancer patients. However, the 5-year survival rates of all PMV patients and RCW patients were comparable to stage IV lung cancer patients. Successful weaning from mechanical ventilation and pneumonia prevention for PMV patients are key factors in improving longterm outcomes in PMV patients.

\section{Abbreviations}


PMV

prolonged mechanical ventilation

RCC

respiratory care center

RCW

respiratory care ward

COPD

chronic obstructive pulmonary disease

CKD

chronic kidney disease

ESRD

end-stage renal disease

CHF

congestive heart failure

CRAB

carbapenem-resistant Acinetobacter baumannii

APACHE II

Acute Physiology, Age, Chronic Health Evaluation II

SAPS

Simplified Acute Physiology Score

\section{Declarations}

\section{Acknowledgments:}

The lung cancer data collected by Buddhist Dalin Tzu Chi hospital Cancer Center, Mr. Yu-Hui Huang.

\section{Funding:}

There was no funding in this study.

\section{Authors' contributions:}

$\mathrm{CH}$ designed the study, collected the data, analyzed the data, wrote the manuscript , and reviewed the manuscript.

\section{Ethics approval and consent to participate}

The project was approved by Buddhist Dalin Tzu Chi general hospital research ethics committee. (Approved IRB No.: B10802009)

Consent for publication: 
Not applicable.

\section{Competing interests:}

The authors declare that they have no competing interests.

\section{Consent}

Written informed consent was obtained from the patient

for publication of this case report and any accompanying images.

\section{References}

1.Bureau of National Health Insurance: Prospective Payment System of Ventilator Dependents Managed Care Demonstration. [ http://www.nhi.gov.tw/nhi/index.aspx].

2. Huang $\mathrm{CH}$. How prolonged mechanical ventilation is a negligent disease in chest medicine - a study of prolonged mechanical ventilation based on six years' experience in Taiwan. Therapeutic Advances in Respiratory Disease. Ready for the publication process by SAGE's production system.

3.Carson SS. Outcomes of prolonged mechanical ventilation. Curr Opin Crit 2006;12:405-11.

4.Damuth E, Mitchel JA, Bartock JL, et al. Long-term survival of critically ill patients treated with prolonged mechanical ventilation: a systematic review and meta-analysis. The Lancet Respir Med 2015;7(3): 544-53.

5.Dettmer MR, Damuth E, Zarbiv S, Mitchell J, Bartock, JL, Trzeciak S. Prognostic Factors for Long-Term Mortality in Critically III Patients Treated With Prolonged Mechanical Ventilation: A Systematic Review. Crit Care Med 2017;45:69-74

6.Stoller JK, Xu M, Mascha E, Rice R. Long-term Outcomes for Patients Discharged From a Long-term HospitalBased Weaning Unit. CHEST 2003; 124:1892-9.

7.Pilcher DV, Bailey MJ, Treacher DF et al. Outcomes, cost and long term survival of patients referred to a regional weaning centre. Thorax 2005; 60:187-92.

8.Scheinhorn DJ, Hassenpflug MS, Votto JJ et al. Post-ICU Mechanical Ventilation at 23 Long-term Care Hospitals: A Multicenter Outcomes Study. Chest 2007; 131:85-93.

9.Bigatello LM, Stelfox, HT, Berra L, Schmidt U, Gettings EM. Outcome of patients undergoing prolonged mechanical ventilation after critical illness. Crit Care Med 2007; 35:2491-7.

10.Cox CE, Martinu, T, Sathy SJ, Clay AS, Chia J, Gray AL et al. Expectations and Outcomes of Prolonged Mechanical Ventilation. Crit Care Med. 2009; 37(11): 2888-94. DOI:10.1097/CCM.0b013e3181ab86ed. 
11.Carson SS, Kahn JM, Hough CL, Seeley EJ, White DB Douglas IS et. al. A multicenter mortality prediction model for patients receiving prolonged mechanical ventilation. Crit Care Med. 2012; 40(4): 1171-6. DOI:10.1097/CCM.0b013e3182387d43.

12.Rose L, Fraser IM. Patient characteristics and outcomes of a provincial prolonged-ventilation weaning centre: A retrospective cohort study. Can Respir J 2012;19(3):216-20.

13.Lin, MS, Yan, YH, Wang, JD, Lu HM. Chen LK, Hung MC et.al. Improved Survival for an Integrated System of Reduced Intensive Respiratory Care for Patients Requiring Prolonged Mechanical Ventilation. Respir Care 2013;58(3):517-24.

14. Hung CT, Lin JW, Ruan SY, Chen CY, Yu CJ. Preadmission tracheostomy is associated with better outcomes in patients with prolonged mechanical ventilation in the postintensive care respiratory care setting. J of Formosan Med Associ 2016 xx, 1e8. http://dx.doi.org/10.1016/j.jfma.2016.05.005.

15.Schönhofer B, Euteneuer S, Nava S, Suchi S, Köhler D. Survival of mechanically ventilated patients admitted to a specialised weaning centre. Intensive Care Med 2002; 28:908-916. DOI 10.1007/s00134002-1287-5.

16.Su J, Lin CY, Chen PJ, Lin FJ, Chen SK, Kuo HT. Experience with a step-down respiratory care center at a tertiary referral medical center in Taiwan. J of Crit care 2006;21:156-61.

https://doi.org/10.1016/j.jcrc.2005.10.004

17.Davies MG, Quinnell TG, Oscroft NS, Clutterbuck SP, Shneerson JM, Smith IE. Hospital outcomes and long-term survival after referral to a specialized weaning unit. British Journal of Anaesthesia, 2017;118: 563-69.

18.Jubran A, Grant BJ, Duffner LA, Collins EG, Lanuza DM, Hoffman LA, et al. Long-term outcome after prolonged mechanical ventilation: a long-term acute-care hospital study. Am J Respir Crit Care Med 2019;199:1508-16. DOI: 10.1164/rccm.201806-11310C

19.Chien JY, Hsueh PR, Yu CJ, Yang PC. The evolution of drug-resistant microorganisms in patients with prolonged mechanical ventilation. Am J Infect Control 2009;37:231-6.

https://doi.org/10.1016/j.ajic.2008.05.016

20.Chan MC, Chiu SK, Hsueh PR, Wang, NC, Wang CC, Fang CT. Risk Factors for Healthcare-Associated Extensively Drug-Resistant Acinetobacter baumannii Infections: A Case-Control Study. PLOS ONE 2014;2014:9:e89973. . DOl:10.1371/journal.pone.0085973

21.Aboussouan LS, Lattin CD, Kline JL. Determinants of Long-Term Mortality after Prolonged Mechanical Ventilation. Lung;2008 186:299-306. DOI 10.1007/s00408-008-9110-x

\section{Figures}




\section{YEARS MORTALITY RATE}

$\longrightarrow$ all PMV patients $\quad-$ RCW patients $\quad \longrightarrow$ Discharged PMV patients

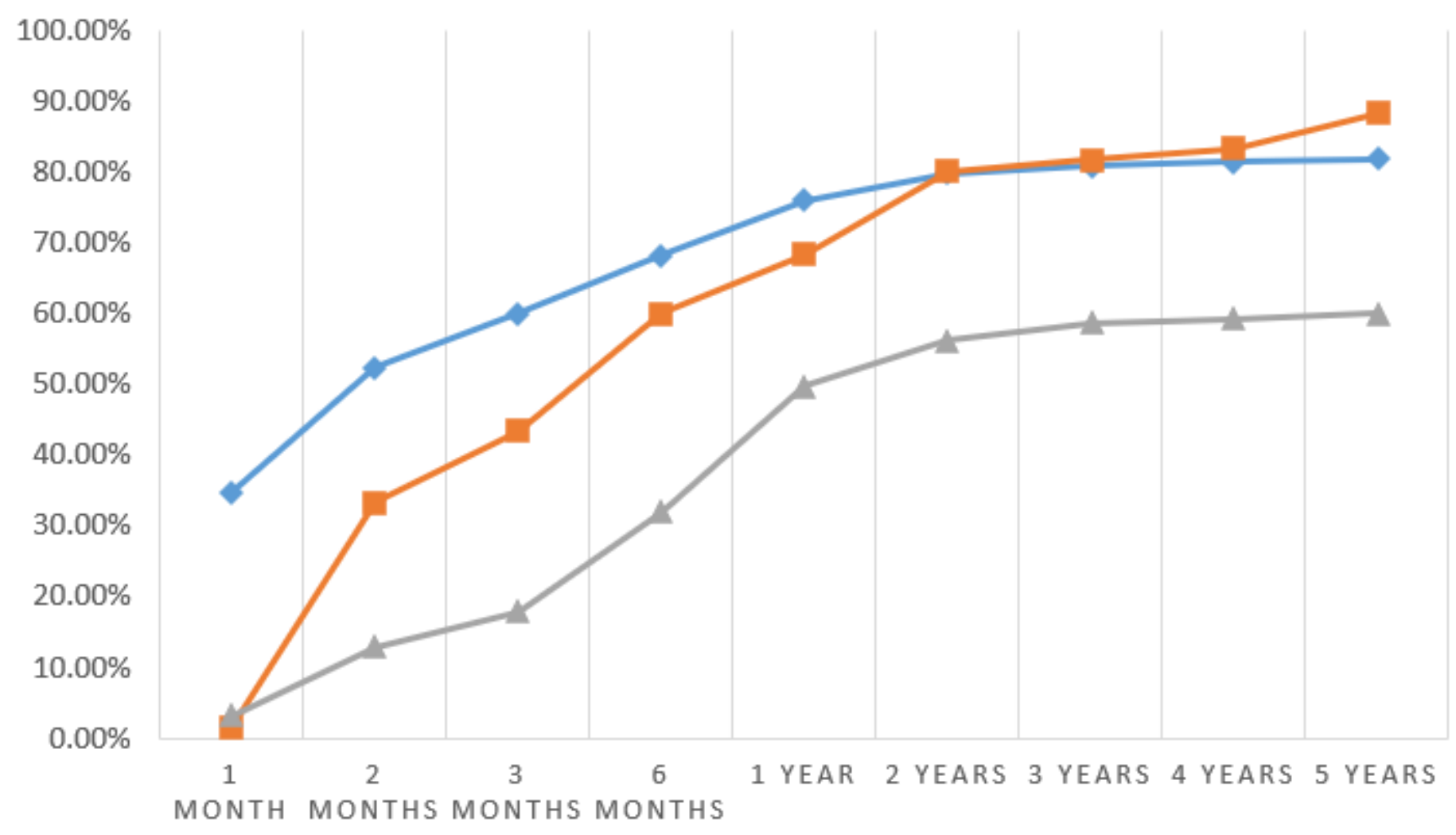

\section{Figure 1}

The five years mortality rate of all PMV patients, RCW patients and Discharged PMV patient 


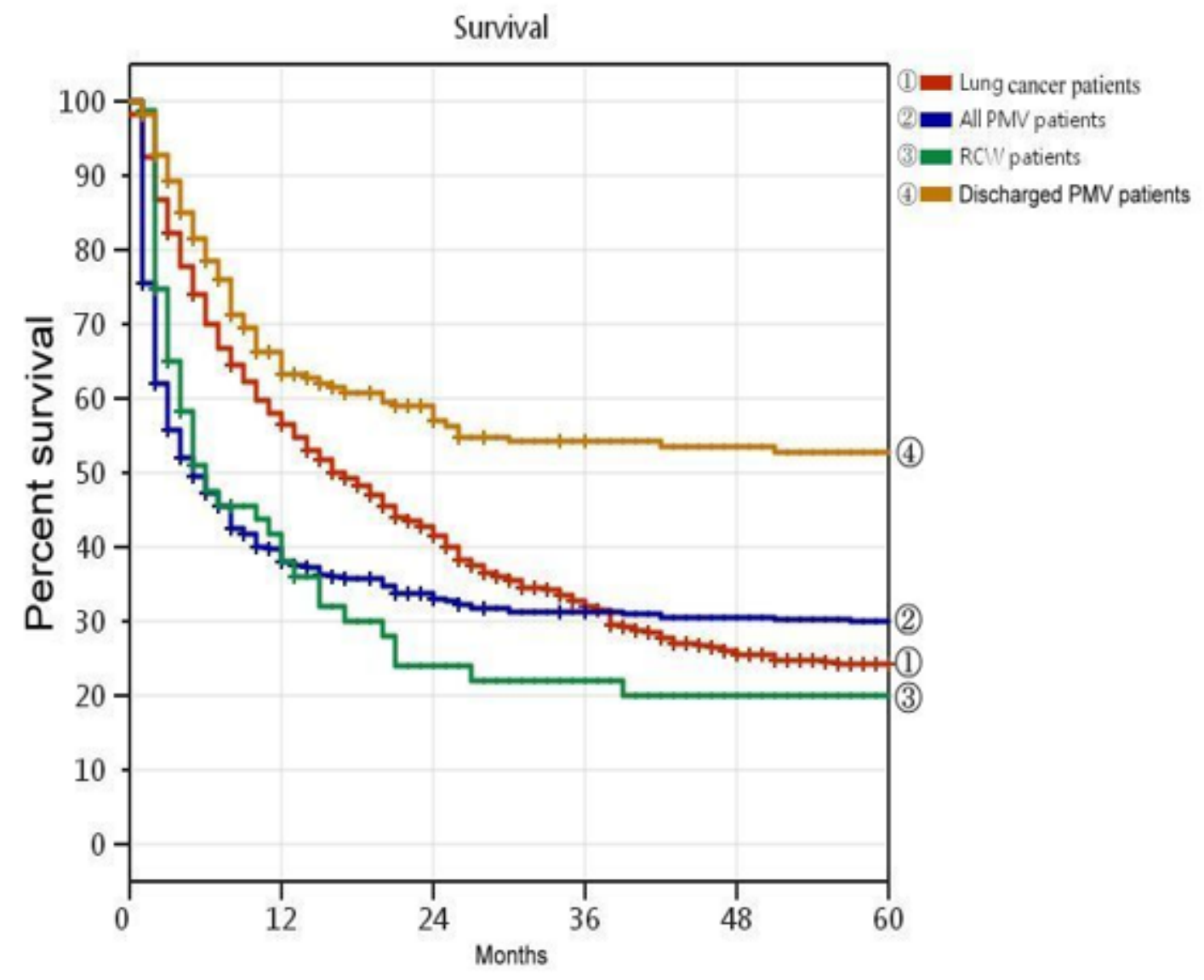

All PMV patients V.S. discharged PMV patients: $\mathrm{P}<0.001, \mathrm{HR}=0.408$.

All PMV patients V.S. RCW patients: $\mathrm{P}=0.653 \mathrm{HR}=0.935$.

discharged PMV patients V.S. RCW patients: $\mathrm{P}<0.001, \mathrm{HR}=2.545$.

All PMV patients V.S. lung cancer patients: $P<0.001, H R=0.732$.

discharged PMV patients V.S. lung cancer patients: $\mathrm{P}<0.001, \mathrm{HR}=1.842$.

RCW patients V.S. lung cancer patients: $\mathrm{P}=0.017, \mathrm{HR}=0.689$.

\section{Figure 2}

The Kaplan-Meier curve of all PMV patients, RCW patients and discharged PMV patient and lung cancer patients 


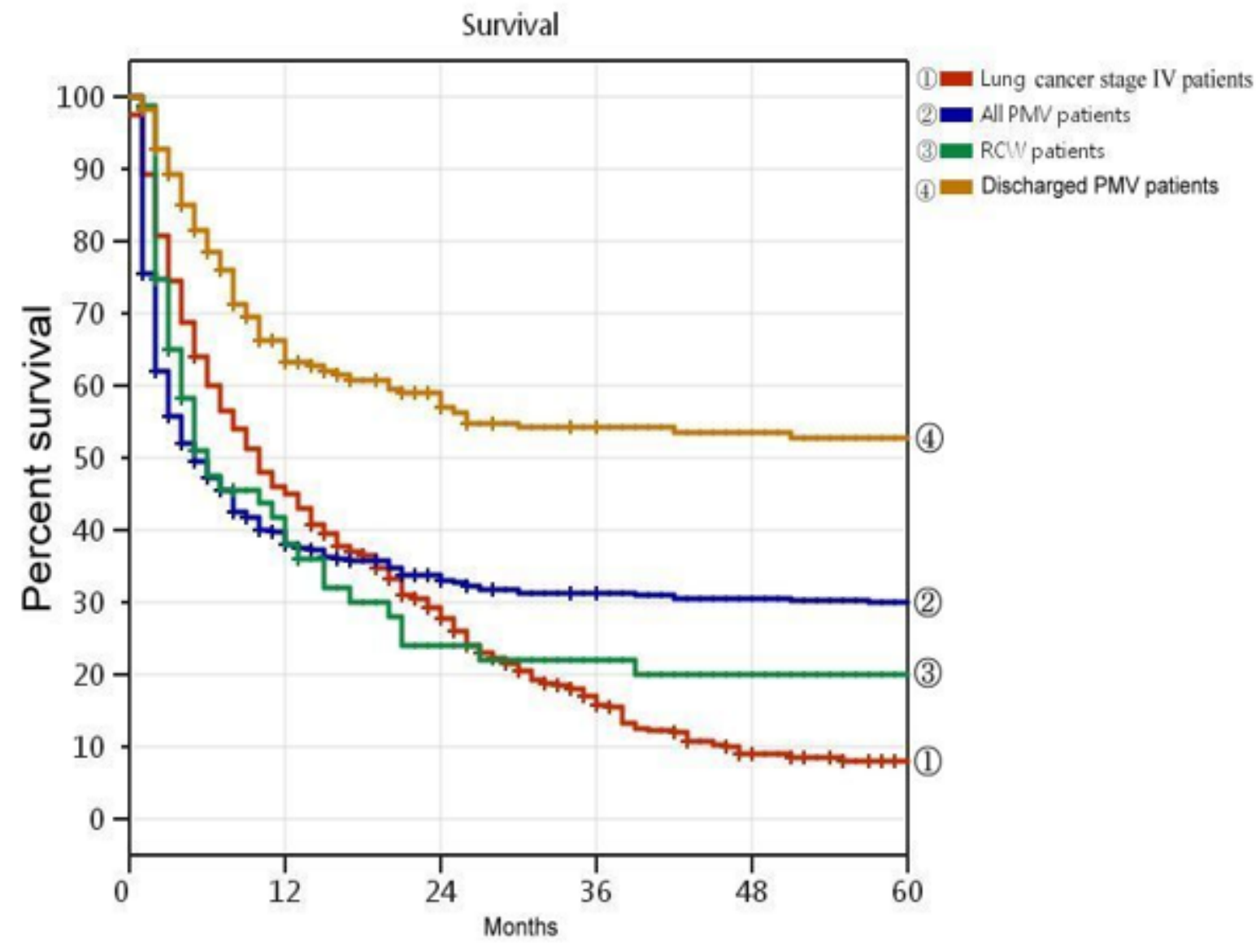

All PMV patients V.S. lung cancer stage IV patients: $\mathrm{P}=0.461, \mathrm{HR}=1.056$.

discharged PMV patients V.S. lung cancer stage IV patients: $\mathrm{P}<0.001, \mathrm{HR}=2.813$.

RCW patients V.S. lung cancer stage IV patients: $\mathrm{P}=0.697, \mathrm{HR}=1.060$.

\section{Figure 3}

The Kaplan-Meier curve of all PMV patients, RCW patients and discharged PMV patient and lung cancer stage IV patients 